\title{
Economic-Effective Multi-Energy Management with Voltage Regulation Networked with Energy Hubs
}

\author{
Pengfei Zhao, Student Member, IEEE, Chenghong Gu, Member, IEEE, Zechun Hu, Senior Member, IEEE, \\ Xin Zhang, Senior Member, IEEE, Xinlei Chen, Ignacio Hernando-Gil, Member, IEEE and Yucheng Ding
}

\begin{abstract}
This paper develops a novel two-stage coordinated volt-pressure optimization (VPO) for integrated energy systems (IES) networked with energy hubs considering renewable energy sources. The promising power-to-gas (P2G) facilities are used for improving the interdependency of the IES. The proposed VPO contains the traditional volt-VAR optimization functionality to mitigate the voltage deviation while ensuring a satisfying gas quality due to the hydrogen mixture. In addition to the conventional voltage regulating devices, i.e., on-load tap changers and capacitor banks, P2G converter and gas storage are used to address the voltage fluctuation problem caused by renewable penetration. Moreover, an effective two-stage distributionally robust optimization (DRO) based on Wasserstein metric is utilized to capture the renewable uncertainty with tractable robust counterpart reformulations. The Wasserstein-metric based ambiguity set enables to provide additional flexibility hedging against renewable uncertainty. Extensive case studies are conducted in a modified IEEE 33-bus system connected with a 20 -node gas system. The proposed VPO provides a voltage-regulated economic operation scheme with gas quality ensured that contributes to high-quality but low-cost multienergy supply to customers.
\end{abstract}

Index Terms -Distributionally robust optimization, energy hub, gas quality management, integrated energy system, two-stage framework.

\section{INTRODUCTION}

Irvent NTEGRATED energy systems (IES) are attracting increasing research attention due to the flexibility to coordinate and contemplate multi-energy infrastructures among each subsystem with promising storage technologies [1-3]. The interdependencies of IES are strengthened by conversion technologies, e.g., power-to-gas ( $\mathrm{P} 2 \mathrm{G})$ and gas turbines.

This work was supported by the the National Natural Science Foundation of China (Nos. 72042018, 71621002) and British Council under Project No. 515761951.

P. Zhao and Z, Cao are with the Institute of Automation, Chinese Academy of Sciences, Beijing, China and School of Artificial Intelligence, University of Chinese Academy of Sciences, Beijing, China. (email: P. Zhao@bath.ac.uk, Zhidong.Cao@ia.ac.cn)

P. Zhao and C.Gu (corresponding author) are with the Department of Electronic \& Electrical Engineering, University of Bath, Bath, UK.(email: P. Zhao@bath.ac.uk; C.Gu@bath.ac.uk).

Z. Hu is with the Department of Electrical Engineering, Tsinghua University, Beijing 100084, China (e-mail: zechhu@ @singhua.edu.cn)

$\mathrm{X}$. Zhang is with the Centre for Energy Systems and Strategy, Cranfield University, Cranfield, UK. (email: xin.zhang@ cranfield.ac.uk).

$\mathrm{X}$. Chen is with the Electrical Engineering Department, Carnegie Mellon University, Pittsburgh, USA. (email: xinlei.chen@sv.cmu.edu)

I. Hernando-Gil is with the ESTIA Institute of Technology, Bidart F 64210, France (e-mail:,i.hernandogil@estia.fr).

Y. Ding is with the China Electric Power Research Institute, Beijing, China. (email: yjs-dyc@epri.sgcc.com.cn)
Although the strong couplings in IES improve the economic performance, it raises some challenges such as: i) the intermittency of renewable energy sources (RES) leads to voltage issues; ii) the uncertainty of RES affects the economic performance of IES and iii) the hydrogen injection via P2G should be ensured with high gas quality. Motivated by the three aforementioned problems, this paper aims to provide a mitigation scheme incorporating voltage regulation, uncertainty modelling and gas quality management.

Volt-VAR optimization (VVO) is the fundamental function for efficiently managing and optimizing the voltage profile within an acceptable range considering system operational constraints. Previous VVO research aims to determine the optimal set of operating voltage regulating devices, e.g., capacitor banks, on-load tap changers (OLTC) and voltage regulators. Paper [4] proposes a voltage regulation algorithm based on flexible alternating current transmission system (FACTS) devices. A multi-objective particle swarm optimization (MOPSO) is applied for reducing the power system costs and ensuring system security. In [5], a MOPSO is applied for optimal power management and design of a hybrid energy system. A coordination algorithm for overcurrent relays and operation strategy is designed in [6] for interconnected power systems. The Pareto-optimal solution is obtained based on MOPSO. Paper [7] develops a novel hybrid multi-objective optimization model for the sizing and placement of renewable energy generations. A non-dominated sorting genetic algorithm is implemented for a two/three-dimensional Pareto optimal set. The high penetration of renewable generation introduces the uncertain renewable fluctuation and technical challenge like voltage variation. Paper [8] applies robust optimization (RO) to a contingency constrained unit commitment model considering uncertain fault outages. The outage probabilities of transmission lines and units are incorporated into the robust uncertainty set. In [9], a multi-band uncertainty set, considering the temporal correlation of renewable generation uncertainty, is developed for a unit commitment model. Paper [10] proposes a robust load-flow in radial and meshed power systems. The nonlinear equation set of load flow is solved via radial basis function artificial neural networks. A novel robust power-flow analysis is presented for balanced and unbalanced microgrids [11]. This model avoids calculating partial derivatives and inverse Jacobian matrix, which is computationally efficient compared with the traditional methods. Existing literature has mainly handled the renewable uncertainty for VVO problems to offset and mitigate the adverse impacts via RO and stochastic 
optimization (SO).

In [12], a multi-timescale VVO is proposed to counteract the voltage fluctuation with separated control on slow and fast voltage regulating devices. The renewable and load uncertainties are handled via SO approach. A distributed multiobjective optimization is proposed in [13] to coordinate fast and slow voltage regulating devices, aiming to mitigate both voltage magnitude and power losses. The PV uncertainty is represented by a scenario-based SO approach. A modified alternating direction method of multipliers is applied to handle the nonconvex optimization model.

Much effort has been focused on the optimization of IES, mainly achieving economic and environmental targets $[14,15]$. A RO model is proposed for an integrated power-gas-heat system in smart districts [16]. This model is demonstrated on a real multi-energy district and real-world physical limitations of energy infrastructures are examined. Paper [17] designs an optimal operation model for a regional IES considering energy price variations. Both system cost and environmental pollutions can be reduced through this optimization model. In [18], an energy sharing framework for multiple interconnected microgrids in an integrated power and heat system is proposed. This model comprehensively optimizes energy generation cost, the trading cost with the utility grid and other microgrids, and discomfort cost. Paper [19] presents a decentralized optimization framework for an integrated power and gas system with networked energy hubs. A distributed algorithm based on Bender's decomposition is used to solve this mixed-integer second-order cone programming problem. In [20], a consumption-based carbon pricing method is combined with an optimization model for IES. Accordingly, energy customers are given proper incentives to use low-carbon energy. Nevertheless, voltage regulation has not been studied in the existing IES operation models to mitigate the system voltage issues.

As an emerging conversion technology, $\mathrm{P} 2 \mathrm{G}$ provides an alternative for promoting RES penetration and provide additional flexibility. The $\mathrm{P} 2 \mathrm{G}$ process is achieved via feeding the surplus renewable generation to electrolysis and produce hydrogen, which can be either transported or stored in gas systems. In [21], a maximum production point tracking strategy to improve the efficiency of $\mathrm{P} 2 \mathrm{G}$ facilities under different operation scenarios. A stochastic operation for a low-carbon micro IES is proposed in [22]. The P2G connected with the wind turbine enables the power-to-hydrogen transformation. Meanwhile, the carbon dioxide-capture-based P2G technology is applied for the eco-friendly IES design. Paper [23] presents a scenario-based optimal strategy for P2G conversion facilities and natural gas generating units. The coordinated operation scheme can provide a high market payoff.

Hydrogen and synthetic natural gas are generated via $\mathrm{P} 2 \mathrm{G}$ electrolyzer, sourced from electric energy. The produced gas mixture can be utilized in gas systems directly or stored via gas storage. Nevertheless, the original gas composition will be inevitably changed due to the additional gas injection. Accordingly, both the security and working performance of gas equipment will be affected [24]. To measure the interchangeability characteristics of different gas components with the comparison of their combustion energy output, Wobbe index (WI) is extensively used. Paper [25] studies the effects under different hydrogen injection levels on natural gas pipeline infrastructures based on WI. It is found that the determinant gas compositions affect the overall hydrogen concentration. The realization of a small-scale renewable hydro methane generator is discussed in [26]. The hydrogen enrichment causes a WI reduction of fuel, which needs to be strictly controlled for safety issues.

The performance of power system operation problems is very sensitive to the renewable uncertainty caused by increasing renewable penetration. The uncertain renewable generation affects i) voltage profile, ii) the optimal operation of $\mathrm{P} 2 \mathrm{G}$ facilities and iii) secure and economic system operation. Most of the existing works account for the uncertainties via RO and SO. However, the ignorance of probabilistic information with RO causes over-conservative solutions with the worst-case orientation. And SO generally assumes the explicit uncertainty distribution based on a large number of scenario representations, which is computationally challenging. As an alternative of RO and SO, the novel distributionally robust optimization (DRO) can address the aforementioned problems based on the ambiguity-averse models considering partial distributional information [27-30].

The effective voltage management through VVO has been extensively studied in the existing research [1-3, 11-13]. The IES integrates a variety of energy vectors to achieve operational effectiveness and the improvement of energy efficiency. However, VVO is ignored in the traditional economic operation of IES, which only focuses on economic performance, which inevitably leads to voltage fluctuation. Overall, in the context of VVO, the existing problems are: i) as a fundamental function in distribution systems, VVO has never been investigated in IES; ii) the emerging P2G with gas storage has never been utilized as voltage regulating devices in the current VVO research and iii) the current IES operation models have never considered the gas quality management schemes. Hence, this paper aims at addressing the above three problems. This paper designs a voltpressure optimization (VPO) including VVO and gas quality management with a two-stage DRO framework in an IES networked with energy hubs. The proposed VPO regulates the voltage magnitude via the traditional voltage regulating devices, i.e., OLTCs, capacitor banks and PV inverters, and P2G with gas storage. To ensure the acceptable gas quality at each gas node, a gas quality management scheme is designed involves adding liquid petroleum gas (LPG) and nitrogen to increase or decrease associated gas quality indices. Renewable uncertainty is handled by the two-stage Wasserstein metric-based DRO. A linear decision rule (LDR)-based solution procedure is developed for solving the two-stage volt-pressure optimization (TS-VPO). The main contributions of this paper are briefly summarized as follows:

- Existing research on IES operation mainly focuses on economic efficiency whilst fails to consider the security and quality of gas. A novel gas quality management in IES is developed to ensure secure and reliable gas system operation.

- IES greatly improves energy efficiency through the coordination and tight couplings among multi-energy infrastructures and converters. However, frequent multi- 
energy interaction also affects the voltage profile of the system. Compared with the existing work purely investigating the voltage management in power systems, this paper develops a VPO model to incorporate the classic VVO problem in IES. P2G facilities and gas storage are used as voltage regulating devices for VVO for the first time.

- When VVO is applied in IES models, it is significantly required to consider gas quality issues. This paper first attempts to investigate the VVO problem and developed gas quality management simultaneously.

- This paper provides a low-carbon and efficient IES networked with energy hubs. The abundant energy conversion technologies in IES networked with energy hubs enable to improve the overall energy efficiency and strengthen the interdependencies among each sub-system.

- Compared with the moment-based DRO approach for handling the data-driven uncertainties with mild robustness, the novel two-stage Wasserstein metric-based DRO approach is proposed. This method further addresses the over-conservative and computational challenging characteristics of RO and SO, respectively.

The remainder of the paper is as follows. The system modelling with technical constraints and objective function of VPO are given in section II. Section III presents the two-stage DRO model with its tractable reformulations. Case studies are given in section IV. And the conclusion is given in V.

\section{MATHEMATICAL FORMULATION OF VPO}

The mathematical formulation of the proposed VPO model is given in this section. The four gas quality indices are firstly described in section A, including WI, specific gravity (SG) and gross calorific value $(\mathrm{GCV})$. The modelling of $\mathrm{P} 2 \mathrm{G}$ and gas storage is given in sections B and C. Sections D-G present the technical constraints of IES. Section $\mathrm{H}$ illustrates the objective function of the two-stage VPO.

Existing papers coordinate voltage regulating devices under different timescales [12, 13, 31], where slow voltage regulating devices (OLTCs and capacitor banks) are dispatched hourly and distributed energy resources (DERs) are dispatched in minute scale. The above multi-timescale models avoid the wearing of mechanical devices caused by frequent adjustment operations and effectively manage the fast variations of DER devices. However, the minute timescale model will increase the decision variables with a larger dimension, thus exponentially increasing the computational burden. Thus this paper utilizes a singletimescale model with hour-timescale.

\section{A. Gas Quality Indices}

In real practice, all gas-fired facilities are designed and equipped according to specific requirements, which include a range of gas quality indices. If the required gas quality standards are not met, a set of issues will arise, such as combustion with poor quality. Gas interchangeability is the main measure to test if the combustion characteristics of one gas resemble other mixture of gases. Two gases are interchangeable when they are substituted with each other without materially changing efficiency, performance and operational safety. The proposed gas quality indices are the explicit expressions of gas interchangeability, which are given in (1)-(3).
In (1), SG is defined as the ratio of the considered gas density to the air density at the same standard temperature and pressure $[24,32]$. This paper regards SG to limit hydrocarbon content, where the density of hydrogen, mixed gas and air are denoted as $\rho_{h y}, \rho_{g}$ and $\rho_{\text {air }} \cdot \varphi_{h y}$ is the hydrogen volume. If hydrocarbon is at a high level, a series of problems will be caused such as engine knock and spontaneous ignition problems.

$$
S G=\frac{\rho_{g}+\left(\rho_{h y}-\rho_{g}\right) \varphi_{h y}}{\rho_{\text {air }}}
$$

When all the considered gas compositions are within the combustion process and meanwhile the gas temperature in the end is equal to the initial gas temperature before the combustion process, the associated gas amount is defined as GCV. GCV is practical for calculating calorific value considering condensation of gas components [33, 34]. Equation (2) is the expression of GCV of mixed gas, where $\Omega_{h y}$ and $\Omega_{g}$ are the GCV of hydrogen and original gas, and $\varphi_{h y}$ is the volume of hydrogen.

$$
\Omega=\Omega_{g}+\left(\Omega_{h y}-\Omega_{g}\right) \varphi_{h y}
$$

WI is a crucial index for measuring gas interchangeability. It is mainly used to compare the combustion output of different gas components [35, 36]. The mixture among gas components is achievable provided that they have similar WI value. Nevertheless, a mild fluctuation of WI is allowable (5-10\% away from the original setpoint). A series of adverse effects will be caused when WI exceeds the acceptable limit, e.g., emergency shutdowns of gas equipment, high emission of greenhouse gas and instability of gas turbines. Equation (3) presents WI expression.

$$
W I=\frac{\Omega}{\sqrt{S G}}
$$

\section{B. Modelling of $P 2 G$}

P2G enables to transform abundant renewable generation to hydrogen and methane through electrolyser. To begin with, the water is split into hydrogen and oxygen, followed by the injection of carbon dioxide in the methanation process. Meanwhile, another part of the produced hydrogen is injected into the gas pipelines directly. The P2G output $G_{n, t}^{h y}$ is given in (4), where $\eta_{e}$ is the efficiency and $P_{n, t}^{P 2 G}$ and $\Omega_{h y}$ is the P2G power injection. Equation (5) shows that the total hydrogen production includes hydrogen used for methanation $\left(G_{n, t}^{h y-m e}\right)$ and direct hydrogen injection $\left(G_{n, t}^{h y}\right)$ to the gas system. The amount of required carbon dioxide and methane production are described in equations (6) and (7), where $\eta_{h y-c a}$ and $\eta_{h y-m e}$ are conversion efficiencies.

$$
\begin{gathered}
G_{n, t}^{h y}=\eta_{e} \frac{P_{n, t}^{P 2 G}}{\Omega_{h y}} \\
G_{n, t}^{h y \_m e}+G_{n, t}^{h y \_}=G_{n, t}^{h y} \\
G_{n, t}^{c a}=\eta_{h y-c a} G_{n, t}^{h y, m e} \\
G_{n, t}^{m e}=\eta_{h y-m e} G_{n, t}^{h y \_m e}
\end{gathered}
$$

\section{Gas Storage System}

The proposed gas storage system (GSS) contains distributed gas storage (DGS) and line pack that provides additional flexibility to the gas system. This paper considers both the original gas and produced gas from P2G can be stored in GSS. DGS enables to store the compressed or liquefied natural gas in tanks. The charging and discharging gas $\left(G_{g s, t}^{c}\right.$ and $\left.G_{g s, t}^{d}\right)$ are 
restricted in (8). Constraints (9) and (10) limit the remaining gas in the DGS, where $E_{g s, t}$ is the remaining gas capacity. Equations (11)-(13) depict the line pack $\vartheta_{l_{g, t}}$ with approximated dynamic characteristics. $f_{l_{g}, t}^{i n i}, f_{l_{g}, t}, P r_{l_{g}, t}^{\text {in i }}$ and $P r_{l_{g}, t}^{\text {ter }}$ are the gas flow and pressure at the initial and terminal nodes. The relationship between the pressure and line pack is given in (11), followed by the gas flow equality constraint defined by line pack in (12). Equation (13) represents the average line flow via the gas flow at the initial and terminal nodes.

$$
\begin{gathered}
0 \leq G_{g s, t}^{\{\}} \leq G_{g s, \max }^{\{\cdot\}},\{\cdot\}=c, d \\
E_{g s, t}=E_{g s, t-1}+G_{g s, t}^{c} \eta_{g s}^{c}-G_{g s, t}^{d} / \eta_{g s}^{d} \\
E_{g s, \min } \leq E_{g s, t} \leq E_{g s, \max } \\
\vartheta_{l_{g, t}}=\Theta_{l_{g}}\left(P r_{l_{g, t}}^{i n i}+P r_{l_{g, t}}^{t e r}\right) \\
f_{l_{g, t}}^{\text {ini }}-f_{l_{g, t}}^{t e r}=\vartheta_{l_{g, t}}-\vartheta_{l_{g, t-1}} \\
f_{l_{g, t}}=\left(f_{l_{g}, t}^{\text {ini }}+f_{l_{g, t}}^{t e r}\right) / 2
\end{gathered}
$$

\section{Power System Constraints}

The power system constraints are presented in (14)-(28). At the substation, the power purchase limit is given in (14) for $P_{s u b, t}^{s}$. The reserve capacity of traditional DGs and gas turbines $\left(r_{i_{e}, t}^{+,-}\right.$and $\left.r_{g t, t}^{+/-}\right)$are constrained in (15) and (16). The generation output of traditional DGs and gas turbines $\left(P_{i_{e}, t}^{s}\right.$ and $\left.P_{g, t}^{s}\right)$ is limited in (17) and (18). Constraint (19) limits the voltage magnitudes for all buses, which is denoted as $V_{b, t}^{s}$. The expression of voltage at the substation is shown in (20) with respect to unit step size of OLTC and tap position, where $V_{\text {subbt }}^{s}$, $V_{\text {sub }}^{r e f}, \delta^{O L T C}$ and $T P_{t}^{\text {s.OLTC }}$ represent the voltage at substation, nominal voltage, size of change for each step in OLTC tap position and tap position of OLTC at time $t$, respectively. Constraint (21) is adopted to avoid the fast wearing process of the transformer with excessive tap operations by the maximum allowing operation times $n T P_{\max }^{\text {OLCC }}$. Constraints (22) and (23) are defined for the reactive power output of PV systems $\omega_{j, t}^{Q, S}$ with regards to the power factor $P F_{p v, \min }$ and active power output $\omega_{j, t}^{P, s}$. The reactive power output of capacitor banks $Q_{c b, t}^{s}$ is constrained in (24), where $u_{c b, t}^{s}$ and $Q_{c b}^{c a p}$ are switch status and capacity of capacitor banks. Constraints (25) and (26) are the DistFlow equation with linearization for distribution networks. This equation is obtained based on the assumption that i) losses are negligible, ii) the voltage at each bus is close to 1.0 p.u. and iii) the voltage at the reference bus is 1.0 p.u. [37-39]. Note that $r_{l_{e}}$ and $x_{l_{e}}$ are resistance and reactance of power line $l_{e}$. The active and reactive power flow are represented by $f_{l_{e}, t}^{a, s}$ and $f_{l_{e}, t}^{r, s}$. And the balancing conditions are given in (27) and (28) for active and reactive power, respectively. $P_{k_{e}, t}$ and $Q_{k_{e}, t}$ are the active and reactive power demand.

$$
\begin{aligned}
& 0 \leq P_{\text {sub, } t}^{s} \leq P_{\text {sub,max }} \\
& 0 \leq r_{\{,, t}^{+} \leq R_{\{,\}}^{+},\{\cdot\}=i_{e}, g t \\
& 0 \leq r_{[\}, t}^{-} \leq R_{\{,-\}}^{-},\{\}=i_{e}, g t \\
& P_{\{\}, t}^{s}+r_{\{\}, t}^{+} \leq P_{\{\}, \text {max }},\{\}=i_{e}, g t \\
& P_{\{\{, \text {min }} \leq P_{\{\{, t, t}^{s}-r_{\{\}, t, t}^{-},\{\cdot\}=i_{e}, g t \\
& V_{b, \min } \leq V_{b, t}^{s} \leq V_{b, \max } \\
& V_{\text {sub }, t}^{s}=V_{\text {sub }}^{r e f}+\delta^{\text {oLTC }} T P_{t}^{s, O L T C} \\
& \sum_{t \in T}\left|T P_{t}^{s, O L T C}-T P_{t-1,}^{s, O L T C}\right| \leq n T P_{\text {max }}^{\text {OLIC }} \\
& 0 \leq \omega_{j, t}^{Q, s} \leq u_{P V} \omega_{j, t}^{P, s}
\end{aligned}
$$

$$
\begin{aligned}
& u_{P V}=\sqrt{\frac{1-P F_{P V, \text { min }}{ }^{2}}{P F_{P V, \text { min }}{ }^{2}}} \\
& Q_{c b, t}^{s}=u_{c b, t}^{s} Q_{c b}^{c a p} \\
& V_{b, t}^{s, i n i}-V_{b, t}^{s, t e r}=\left(f_{l_{e}, t}^{a, s} r_{l_{e}}+f_{l_{e}, t}^{r, s} x_{l_{e}}\right) / V_{0} \\
& 0 \leq f_{l_{e}, t}^{\{\cdot\}, s} \leq f_{l_{e}, \text { max }}^{\{\cdot\}, s}\{\cdot\}=a, r \\
& \sum_{i_{e} \in I_{e}} P_{i_{e}, t}^{s}+\sum_{j \in J} \omega_{j, t}^{P, s}+\sum_{l_{e} \in L_{e}} f_{l_{e}, t}^{a, s, i n i}-\sum_{l_{e} \in L_{e}} f_{l_{e}, t}^{a, s, t e r}+\sum_{g t \in G T} P_{g t, t}^{s} \\
& =\sum_{k_{e} \in K_{e}} P_{k_{e}, t}+\sum_{n \in N} P_{n, t}^{S, P 2 G} \\
& \sum_{i_{e} \in I_{e}} Q_{l_{e}, t}^{s}+\sum_{j \in I} \omega_{j, t}^{Q, s}+\sum_{c b \in C B} Q_{c b, t}^{s}+\sum_{l_{e} \in L_{e}} f_{l_{e}, t}^{r, s, i n i}-\sum_{l_{e} \in L_{e}} f_{l_{e}, t}^{r s, t e r}=\sum_{k_{e} \in K_{e}} Q_{k_{e}, t}
\end{aligned}
$$

\section{E. Gas System Constraints}

Equations (29)-(43) are technical constraints of the gas system. The gas source output $G_{i g, t}^{s}$ is limited in (29). Equations (30) and (31) regulate the gas pressure $P r_{l g, t}^{s}$, where constraint (31) indicates that the inlet gas pressure $P r_{l g, t}^{s, i n i}$ is always larger than the outlet gas pressure $P r_{l g, t}^{s, t e r}$. To define the gas flow $f_{l_{g}, t}^{s}$, constraints (32) and (33) are utilized as the Weymouth gas flow equation, where $\gamma_{l_{g}}$ is the Weymouth equation constant. The gas turbine output $P_{g t, t}^{s}$ is restricted in (34). The proposed gas quality constraints under real conditions are presented in (35)-(37), including GCV, SG and WI. For the sake of gas quality and security, the limitation of gas quality indices is given in (38). In (39), the gas volume deviation is limited between the adjacent time periods under standard temperature and pressure. Constraints (40)-(42) limit the overall gas volume $\varphi_{n, t}^{\text {mix }}$ at each gas node according to [40], where $\Theta$ is the constant in Boyle's law. And the gas balance constraint is presented in (43).

$$
\begin{aligned}
& G_{i_{g}, \min } \leq G_{i_{g}, t}^{S} \leq G_{i_{g}, \max } \\
& \operatorname{Pr}_{l_{g}, \min }^{2} \leq \operatorname{Pr}_{l_{g}, t}^{S^{2}} \leq P r_{l_{g}, \max }^{2} \\
& P r_{l g, t}^{s, i n i} \geq P r_{l_{g}, t}^{s, t e r} \\
& f_{l_{g}, t}^{s, 2}=\gamma_{l_{g}}\left(P r_{l_{g}, t}^{s, i n i^{2}}-P r_{l_{g}, t}^{s, t e r}{ }^{2}\right) \\
& 0 \leq f_{l g, t}^{s} \leq f_{l g, \max } \\
& P_{g t, t}^{s}=c_{g t} f_{l_{g}, g t}^{s} \\
& \Omega_{n, t}^{m i x}=\Omega_{h y}\left(\varphi_{n, t}^{h y, m e}+\varphi_{n, t}^{h y, d}\right)+\Omega_{L P G} \varphi_{n, t}^{L P G}+\Omega_{n i} \varphi_{n, t}^{n i}+\Omega_{m e} \varphi_{n, t}^{m e} \\
& S G_{n, t}^{m i x}=\left[\rho_{h y}\left(\varphi_{n, t}^{h y}+m e+\varphi_{n, t}^{h y-d}\right)+\rho_{L P G} \varphi_{n, t}^{L P G}+\rho_{n i} \varphi_{n, t}^{n i}+\rho_{m e} \varphi_{n, t}^{m e}\right]\left(\varphi_{n, t}^{h y, m e}\right. \\
& \left.+\varphi_{n, t}^{h y, d}+\varphi_{n, t}^{L P G}+\varphi_{n, t}^{n i}+\varphi_{n, t}^{m e}\right) \\
& W I_{n, t}^{\text {mix }}=\Omega_{n, t}^{\text {mix }} / \sqrt{S G_{n, t}^{\text {mix }}} \\
& \{\cdot\}_{\min } \leq\{\cdot\} \leq\{\cdot\}_{\text {max }} \\
& \{\cdot\}=\Omega_{n, t}^{\text {mix }}, S G_{n, t}^{\text {mix }}, W I_{n, t}^{\text {mix }} \\
& -\Delta \varphi_{n, \max }^{\{\cdot\}} \leq \varphi_{n, t}^{\{\cdot\}}-\varphi_{n, t-1}^{\{\cdot\}} \leq \Delta \varphi_{n, \max }^{\{\cdot\}} \\
& \{\cdot\}=h y_{-} m e, h y_{-} d, L P G, n i, m e \\
& \varphi_{n, t}^{h y, m e}+\varphi_{n, t}^{h y, d}+\varphi_{n, t}^{L P G}+\varphi_{n, t}^{n i}+\varphi_{n, t}^{m e}=\varphi_{n, t}^{m i x} \\
& \varphi_{n, \min }^{\operatorname{mix}} \leq \varphi_{n, t}^{\operatorname{mix}} \leq \varphi_{n, \max }^{\operatorname{mix}} \\
& \varphi_{n, t}^{\text {mix }}=\frac{\Theta}{P r_{n, t}} \\
& \sum_{i_{g} \in l_{g}} G_{i_{g}, t}^{s}+\sum_{n \in N} G_{n, t}^{s, h y}+\sum_{l_{g} \in L_{g}} f_{l_{g}, t}^{s, i n i}-\sum_{l_{g} \in L_{g}} f_{l_{g}, t}^{s, t e r} \\
& =\sum_{k_{g} \in K_{g}} G_{k_{g}, t}+\sum_{l_{g} \in L_{g}} f_{l_{g}, g t, t}^{s}
\end{aligned}
$$

\section{F. Energy Hub Constraints}

The energy hub considered in this paper is equipped with combined heat and power (CHP), gas furnace (GF), ground source heat pump (GSHP) for energy conversion and an energy storage system for storing the excessive energy. The detailed 


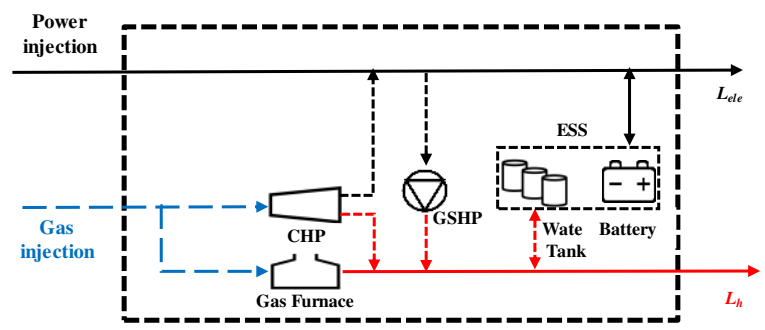

Fig. 1. The proposed energy hub model.

energy hub structure is given in Fig. 1. The energy storage system contains a battery storage and a heat storage. Constraint (44) presents the expression of the energy conversion of GSHP and GF, i.e., $P_{C O P, t}^{s, o}$ and $P_{G F, t}^{s, o}$. Equations (45) and (46) show the power and heat output of CHP $\left(P_{c p^{,}, t}^{s, o}\right.$ and $\left.P_{c p^{h}, t}^{s,}\right)$. Constraint (47) limits the input of CHP, GF and GSHP $\left(P_{c p, t}^{i}, P_{C O P, t}^{i}\right.$ and $\left.P_{G F, t}^{i}\right)$. The hourly charging and discharging power and heat for battery and water tank are given in (48) for $P_{B S, t}^{s, c h}, P_{B S, t}^{s, d c h}, P_{H S, t}^{s, c h}$ and $P_{H S, t}^{\text {s,dch }}$. The remaining power and heat energy $\left(E_{B S, t}^{s}\right.$ and $\left.E_{H S, t}^{s}\right)$ is limited in (49) and (50). Equation (51) is the coupling constraint for energy hub for maintaining the balancing condition.

$$
\begin{aligned}
& P_{\{;, t, t}^{s, o}=\eta_{\{,\}]\{\}, t, t}^{s, i},\{\}=C O P, G F \\
& P_{c p^{e}, t}^{s, o}=\eta_{c p^{e}} P_{c p, t}^{s, i} \\
& P_{c p^{h}, t}^{s, o}=\eta_{c p^{h}} P_{c p, t}^{s, i} \\
& P_{\{\cdot\} \text {,min }}^{i} \leq P_{\{,\}, t}^{i} \leq P_{\{\cdot\}, \text { max }}^{i},\{\cdot\}=c p, C O P, G F \\
& P_{\{\}, \text {min }}^{s, c h / d c h} \leq P_{\{\}, t}^{s, c h / d c h} \leq P_{\{\}, \text {max }}^{s, c h / d c h},\{\}=B S, H S \\
& E_{\{\}, t}^{s}=E_{\{\}, t-1}^{s}+\sum_{1}^{t} P_{\{\}, t, t}^{s, c h} \eta_{\{\}}^{c h}-P_{\{\}, t}^{s, d c h} / \eta_{\{\}\}}^{d c h},\{\}=B S, H S \\
& E_{\{\}, \min } \leq E_{\{\}, t}^{S} \leq E_{\{\}, \max },\{\cdot\}=B S, H S \\
& {\left[\begin{array}{l}
L_{e, t}+P_{B S, t}^{S} \\
L_{h, t}+P_{H S, t}^{S}
\end{array}\right]=} \\
& {\left[\begin{array}{cc}
1-v_{e, t}^{s} & v_{g, t}^{s} \eta_{C H P^{e}}\left(1-v_{e, t}^{s}\right) \\
v_{e, t}^{s} \eta_{C O P} & v_{g, t}^{s}\left(\eta_{C H P^{h}}+\eta_{C H P} v_{e, t}^{s} \eta_{C O P}+\eta_{G F}-v_{g, t}^{s} \eta_{G F}\right)
\end{array}\right] \times\left[\begin{array}{l}
f_{l_{e}}^{s, i n j} \\
f_{l_{g, t}}^{s, i n j}
\end{array}\right]}
\end{aligned}
$$

\section{G. Real-Time System Constraints}

The endogenous renewable uncertainty and variability are realized after the first-stage decision making. And the real-time corrective VPO can be implemented for adjustment on voltage regulation, gas quality control and redispatch of generators. Moreover, load shedding is scheduled for ensuring the security of the overall system. The real-time regulation of operation schemes of traditional DGs and gas turbines are given in (52). Constraint (53) presents the load shedding limits for power and gas systems $\left(P_{k_{e}, t}^{l s}\right.$ and $\left.P_{k_{g}, t}^{l s}\right)$. Finally, the real-time balancing conditions of power and gas systems are shown in (54)-(56). Noted that the rest of the second-stage constraints are not given, but are the same as the first-stage constraints presented in section II-C, D and E when superscript ' $s$ ' is replaced by ' $r e$ '.

$$
\begin{aligned}
& P_{\{,\}, t}^{r e}-r_{\{,\}, t}^{-} \leq P_{\{,\}, t}^{r e} \leq P_{\{,\}, t}^{r e}+r_{\{,\}, t}^{+},\{\cdot\}=i_{e}, g t \\
& 0 \leq P_{\{l, t, t}^{l s} \leq P_{\{\cdot\}, \max }^{l s},\{\}=k_{e}, k_{g}
\end{aligned}
$$

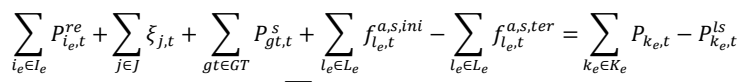

$$
\begin{aligned}
& +\sum_{n \in N} P_{n, t}^{r e, P 2 G}
\end{aligned}
$$

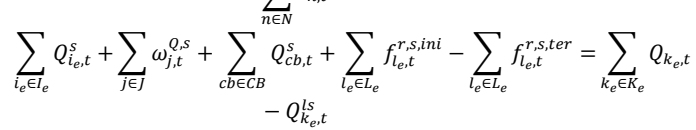

$$
\sum_{i_{g} \in I_{g}} G_{g_{g}, t}^{r e}+\sum_{l_{g} \in L_{g}} f_{l_{g}, t}^{r e, i n i}-\sum_{l_{g} \in L_{g}} f_{g_{g}, t}^{r, t e r}=\sum_{k_{g} \in K_{g}} G_{k_{g}, t}-G_{k_{g}, t}^{l e}+\sum_{l_{g} \in L_{g}} f_{l_{g} g, t}^{r e}
$$

\section{H. Objective Function}

The objective function of the first and second stages are presented in (57) and (58), respectively. In (57), the minimization of voltage deviation and system operation cost is proposed. The cost coefficient of voltage regulation, gas quality management, gas storage depreciation, substation power purchase, power generation, natural gas source generation and reserve capacity are denoted by $\pi_{v}, \lambda_{N}, \lambda_{L P G}, \lambda_{s u b}, \lambda_{g s}, \lambda_{i_{e}}^{a}, \lambda_{i_{e}}^{b}, \lambda_{i_{e}}^{c}$, $\lambda_{i_{g}}, \lambda_{i_{e}}^{+}$and $\lambda_{i_{e}}^{-}$. The first term transforms the voltage deviation to monetary loss. The second and third terms depict the purchase and injection cost of nitrogen and LPG for maintaining satisfied gas quality indices. The power purchase from the upper-level market is given in the fourth term. The fifth term represents the depreciation cost of DGS. And the rest of (57) shows the generation and reserve cost of traditional DGs and gas turbines. Equation (58) presents the second-stage objective function including the voltage deviation $\operatorname{cost}\left(\pi_{v}||_{b, t}^{\text {re }}-V_{b}^{\text {ref }} \mid\right)$, the regulation cost for re-dispatching generators $\left(\lambda_{N} \varphi_{n, t}^{r e n i}+\lambda_{L P G} \varphi_{n, t}^{r e, L P G}+\right.$ $\left.\lambda_{\text {sub }} P_{\text {sub,t }}^{r e}+\lambda_{j}^{r e}\left|\omega_{j, t}^{s}-\xi_{j, t}\right|+\lambda_{i_{e}}^{r e}\left|P_{i_{e, t}}^{s}-P_{i_{e}, t}^{r e}\right|+\lambda_{i_{g}}^{r e}\left|P_{i_{g}, t}^{s}-P_{i_{g}, t}^{r e}\right|\right)$ and load shedding $\left(\lambda_{k_{e}}^{l s} P_{k_{e}, t}^{l s}+\lambda_{k_{g}}^{l s} P_{k_{g}, t}^{l s}\right)$.

$$
\begin{aligned}
& \Gamma_{1}=\min \sum_{i_{e} \in I_{e}, i_{g} \in I_{g}, t \in T} \pi_{v}\left|V_{b, t}^{s}-V_{b}^{r e f}\right|+\lambda_{N} \varphi_{n, t}^{s, n i}+\lambda_{L P G} \varphi_{n, t}^{s, L G G} \\
& +\lambda_{s u b} P_{s u b, t}^{s}+\lambda_{g s}\left(G_{g s, t}^{c}+G_{g s, t}^{d}\right)+\lambda_{i_{e}}^{a} P_{i_{e}, t}^{s}{ }^{2} \\
& +\lambda_{i_{e}}^{b} P_{i_{e, t}}^{s}+\lambda_{i_{e}}^{c}+\lambda_{i_{g}} P_{i_{g}, t}^{s}+\lambda_{i_{e}}^{+} r_{i_{e, t}}^{+}+\lambda_{i_{e}}^{-} r_{i_{e, t}}^{-} \\
& \Gamma_{2}=\min \sum_{i_{e} \in \epsilon_{e}, i_{g} \in I_{g}, t \in T, k_{e} \in K_{e}, k_{g} \in K_{g}} \pi_{v}\left|V_{b, t}^{r e}-V_{b}^{r e f}\right|+\lambda_{N} \varphi_{n, t}^{r e, n i} \\
& +\lambda_{L P G} \varphi_{n, t}^{r e, L P G}+\lambda_{\text {sub }} P_{\text {sub }, t}^{r e}+\lambda_{j}^{r e}\left|\omega_{j, t}^{s}-\xi_{j, t}\right| \\
& +\lambda_{i_{e}}^{r e}\left|P_{i_{e}, t}^{s}-P_{i_{e}, t}^{r e}\right|+\lambda_{i_{g}}^{r e}\left|P_{i_{g}, t}^{s}-P_{i_{g}, t}^{r e}\right|+\lambda_{k_{e}}^{l s} P_{k_{e}, t}^{l s} \\
& +\lambda_{k_{g}}^{l s} P_{k_{g}, t}^{l s}
\end{aligned}
$$

\section{MethodOLOGY}

The solution approach of the two-stage DRO is given in this section. In Fig. 2, the method illustration is presented including the description of the following four subsections.

\section{A. Compact Matrix Formulation}

For the clear presentation and notation brevity, the original problem is given as a compact matrix formulation. The firststage problem is given in (59) and (60), where the first-stage variables are represented by vector $x$. Objective (59) represents (57)-(58) and constraint (60) represents (4)-(51) in the first stage. The second-stage objective $Q(x, \xi)$ is the wait-and-see adaptive objective given the here-and-now decision $x$.

$$
\begin{gathered}
\min _{x \in X} c^{T} x+\sup _{\mathbb{P} \in \Omega} E_{\mathbb{P}}[Q(x, \xi)] \\
\text { s.t. } A x \leq b, x \in \mathbb{R}^{V_{1}}, b \in \mathbb{R}^{C_{1}}, A \in \mathbb{R}^{C_{1} \times V_{1}}
\end{gathered}
$$

The second-stage problem is shown in (61) and (62), and $y$ denotes the second-stage variables. Constraints (4)-(51) with superscript ' $r e$ ' and (52)-(56) are summarized as (62). In (63), vector $h(\xi)$ is composed of the constant vector $h^{0}$ and uncertain vector $h_{i}^{\xi}$.

$$
\begin{gathered}
Q(x, \xi)=\min _{y} f^{\prime} y, y \in \mathbb{R}^{V_{2}} \\
\text { s.t. } B x+C y \leq h(\xi), y \in \mathbb{R}^{V_{2}}, h \in \mathbb{R}^{C_{2}}, B \in \mathbb{R}^{C_{2} \times V_{1}}, \\
C \in \mathbb{R}^{C_{2} \times V_{2}}, D \in \mathbb{R}^{C_{2} \times i} \\
h(\xi)=h^{0}+h_{i}^{\xi} \xi_{i}
\end{gathered}
$$




\section{B. Wasserstein Distance-Based Ambiguity Set}

According to the data-driven setting with the empirical distribution $\widehat{\mathbb{P}}=1 / S \sum_{s \in S} \delta_{\widehat{\xi}}$, the Wasserstein metric between the candidate and empirical distributions is given in (64) [41]. In Fig. 3, the figure presentation shows that Wasserstein metirc is used to measure the similarity of two distributions. The random variables in the candidate and empirical distributions are denoted as $\xi$ and $\xi^{\dagger}$, respectively. The distance metric is represented by $\rho\left(\xi, \xi^{\dagger}\right)$.

$$
d(\mathbb{P}, \widehat{\mathbb{P}})=\inf E_{\mathbb{Q}}\left[\rho\left(\xi, \xi^{\dagger}\right)\right], \xi \sim \mathbb{P}, \xi^{\dagger} \sim \widehat{\mathbb{P}}
$$

The ambiguity set considering the Wasserstein distance is presented in (65), where is the radius of the ball set. The set of all the possible distributions is denoted as $\boldsymbol{P}$.

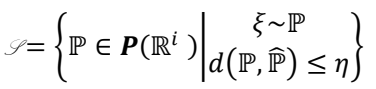

The explicit conditional Wasserstein-based ambiguity set is given in (66), where the scenarios are distinguished by $\tilde{s}$, representing the support of $\xi$ is different based on different scenarios. The ambiguity set in (66) ensures i) the uncertain variables $\xi, \varphi$ and $\tilde{s}$ are within the distribution; ii) the expectation of uncertain variable $\xi$ is $\mu_{s}$; iii) the auxiliary variable $\varphi$ is used to ensure limited the distribution distance and iv) $\xi$ and $\varphi$ are limited within the lifted support set $\Xi$.

$$
\Omega=\left\{\begin{array}{l|c}
((\xi, \varphi), \tilde{s}) \sim \mathbb{P} \\
\mathbb{P} \in \boldsymbol{P}\left(\mathbb{R}^{i} \times \mathbb{R}^{j}\right) & \begin{array}{c}
(\xi) \mid s=S]=\mu_{s} \\
E_{\mathbb{P}}[\xi \mid \tilde{s} \in S] \leq \eta_{s} \\
\Xi=\left\{(\xi, \varphi) \in \mathbb{R}^{i} \times \mathbb{R}^{j}: G x+H y \leq r\right\} \\
\mathbb{P}[(\xi, \varphi) \mid \tilde{s} \in S]=1 \\
\mathbb{P}[\tilde{s} \in S]=1
\end{array}
\end{array}\right\}
$$

\section{Approximation via Linear Decision Rule}

Equation (67) is obtained as is equivalent to $Q(x, \xi)$, where $y(\xi)$ is the adaptive recourse function as shown in (68). Determining the worst-case expectation is generally intractable since all the possible realizations pertaining to the uncertainties are involved [42]. Employing the LDR in (69) can address the problem [43], which approximates $y(\xi)$ by linear affine functions of $\xi$ and $\varphi$.

$$
\begin{gathered}
Q(x, \xi)=\sup _{\mathbb{P} \in \Omega} E_{\mathbb{P}}[Q(x, \xi)]=\sup _{\mathbb{P} \in \Omega} E_{\mathbb{P}}\left[f^{\prime} y(\xi)\right] \\
y(\xi) \in \arg \min \left\{f^{\prime} y: B x+C y \leq h(\xi)\right\}, y \in \mathbb{R}^{V_{2}}, h \in \\
\mathbb{R}^{C_{2}}, B \in \mathbb{R}^{C_{2} \times V_{1}}, C \in \mathbb{R}^{C_{2} \times V_{2}}, D \in \mathbb{R}^{C_{2} \times i} \\
y_{n}(\xi, \varphi)=y_{n}^{0}+\sum_{\xi \in \tilde{\xi}} y_{n}^{\xi} \xi+\sum_{\varphi \in \widetilde{\varphi}} y_{n}^{\varphi} \varphi
\end{gathered}
$$

The approximation of function $Q(x, \xi)$ can be obtained when the recourse decision $y(\xi)$ is replaced by the LDR expression in (70), which is denoted as $Q_{L D R}(x, \xi)$.

$$
\begin{aligned}
& Q_{L D R}(x, \xi, \varphi, \tilde{s})=\min \sup _{\mathbb{P} \in \Omega} E_{\mathbb{P}}\left[f^{\prime} y(\xi, \varphi, \tilde{s})\right] \\
& \text { s.t. } B x+C y(\xi, \varphi, \tilde{s}) \leq h(\xi), \forall(\xi, \varphi) \in \Xi
\end{aligned}
$$

\section{Dual Reformulation and Robust Counterpart}

To convert the original 'min sup' framework of the second stage into 'min' and thus mixed with the first-stage objective, a dual reformulation is made [44] in (72)-(75), where $\psi$ and $\lambda$ are dual variables.

$$
\begin{gathered}
Q_{L D R}=\min \tau+\psi \eta_{s}+\lambda \mu_{s} \\
\text { s.t. } \tau+\xi^{\prime} \lambda+\varphi^{\prime} \psi \geq f^{\prime} y(\xi, \varphi, \tilde{s}), \forall(\xi, \varphi) \in \Xi \\
B x+C y(\xi, \varphi, \tilde{s}) \leq h(\xi), \forall(\xi, \varphi) \in \Xi
\end{gathered}
$$

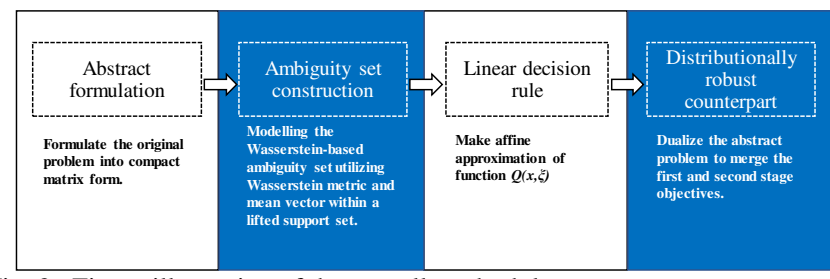

Fig. 2. Figure illustration of the overall methodology.

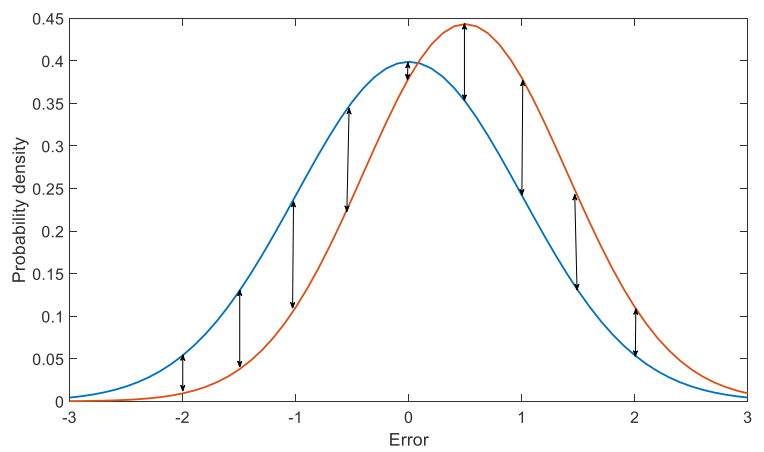

Fig. 3. Wassertein metric between two probability distributions.

$$
\psi \geq 0, \psi \in \mathbb{R}^{j}, \tau \in \mathbb{R}, \lambda \in \mathbb{R}^{i}
$$

The reformulated (72)-(75) is a robust linear program, which can be written as the robust counterpart in (77)-(84).

$$
\begin{gathered}
Q_{L D R}=\min \tau+\psi \eta_{s}+\lambda \mu_{s} \\
\text { s.t. } \tau-f^{\prime} y^{0 s}+\chi_{0}^{\prime} r \geq 0 \\
\chi_{0 s}^{\prime} G_{s i}=\sum_{n} q_{n} y_{n i}^{\xi s}-\lambda_{i}, \forall i \in I, \forall s \in S \\
\chi_{0 s}^{\prime} H_{s j}=\sum_{n} q_{n} y_{n j}^{\varphi s}-\psi_{j}, \forall j \in J, \forall s \in S \\
\chi_{m s}^{\prime} G_{s i}=\sum_{n}^{n} C_{m n} y_{n i}^{\xi s}-h_{m i}^{\xi}, \forall i \in I, \forall s \in S \\
\chi_{m s}^{\prime} H_{s j}=\sum_{n} C_{m n} y_{n j}^{\varphi s}, \forall j \in J, \forall s \in S \\
B_{m}^{\prime} x+C_{m}^{\prime} y^{0 s}-h_{m}^{0}+r^{\prime} \chi_{m s}, \forall s \in S
\end{gathered}
$$

The new dual variables are represented as $\chi_{0}$ and $\chi_{m}$, respectively. Accordingly, the tractable approximation of the original TS-VPO is derived in (76)-(83).

\section{CASE STUdies}

This section presents the case studies to verify the effectiveness of the proposed TS-VPO on a 33-bus-20-node IES networked with energy hubs. The test system contains two gas turbines and $\mathrm{P} 2 \mathrm{G}$ facilities for the power-gas interconnection [45]. Two DGSs are connected with P2G facilities for storing excessive gas. Two energy hubs are connected with buses 8 and 21 , where the explicit structure of energy hubs are shown in Fig. 1. The power system contains 4 PV systems, 3 traditional DGs as well as 7 capacitor banks. The rated capacity of each capacitor bank and PV system are set as 400kVar and 360kVA. TABLE I describes the 6 cases for testing the performance of TS-VPO. The radius of the Wasserstein ball is $1 \%$ of the upper bound of random variables.

\section{A. Studies on Voltage Management}

In Fig. 4, the voltage profiles of cases 1-4 are given. The blue solid curve represents the voltage at all buses for 24 hours and the red dotted curve represents the mean voltage value. In Fig. 
4 (a), the voltage magnitude ranges from 0.96 p.u. to 1.05 p.u. The voltage at bus 1 is the highest, followed by the decrease across the main branch till bus 18 . The lowest voltage at the main branch reaches 0.97 p.u. at bus 18 . Another decreasing trend occurs at buses 19-33. At bus 33, the voltage reaches 0.96 p.u.. In Fig. 4 (b), compared with case 1, the voltage profile of case 2 ranges between 0.97 p.u. and 1.03 p.u.. The gas-to-power (G2P) is removed from the modelling and thus results in less voltage fluctuation. Case 3 only considers G2P and the voltage magnitude is generally larger than both cases 1 and 2 since the power conversion and hydrogen production are not considered. In particular, the voltage decreasing rate from bus 6 to 18 is slower than that of case 1 . When the interconnection between power and gas systems is not considered, the voltage profile shows a similar voltage scheduling to case 1 .

The reactive power output scheduling for PV systems of cases 1 and 5 are shown in Figs. 5 and 6. The overall reactive power output in case 1 shows a smooth scheduling curve compared with case 5, which ranges between 0 and 0.3 MVar. The PV reactive power output remains the same at buses 3 and 6 over the entire time horizon, i.e., 0. 13MVar and $-0.13 \mathrm{MVar}$, respectively. At bus 26, the reactive power output is sensitive to load demand variation, which changes frequently to absorb and compensate the reactive power. The PV reactive power shows a dramatic drop and rise between 2:00 and 7:00 in case 5, particularly at bus 6 . On the contrary, buses 3,11 and 25 yield similar reactive power output at each hour.

The remaining capacity of DGS at nodes 10, 12 and 14 are presented in Fig. 7. The DGS at nodes 10 and 12 have more frequent usage than node 14. At node 10, the DGS is charging before 4:00, followed by a standby status between 4:00 and 8:00. Then it is generally discharging until 21:00. The last serval hours witness another charging process to meet the remaining capacity equal to the initial level. The voltage at bus 11, however, generally shows a flat trend compared with the DGS scheduling at node 10 . The voltage is adjusted to remain around 1.00 p.u. when DGS is extensively utilized in the charging process to store the excessive power injection and vice versa. On the contrary, since bus 3 is vital for distributing power on the main and sub-branches, the voltage fluctuation at bus 3 is distinct and cannot be effectively mitigated by the DGS, which results in the slight usage of DGS at node 14.

\section{B. Studies on Economic Performance}

TABLE II shows the results of the operation cost of two stages. The operation cost case 2 is the highest among all cases, i.e., $\$ 166766$. In contrast, case 6 results in the lowest operation cost $(\$ 139072)$. The benchmark case 1 yields $\$ 126244$ and $\$ 30458$ at the first and second stages, respectively. The $\$ 10064$ higher operation cost of case 2 is due to the disconnection of G2P supply. Compared with case 2, the operation cost of case 3 decreases, implying the advantage of GSP over P2G on the minimization of system operation cost. In case 4, there are no interconnections for power and gas systems. And the operation cost is $0.3 \%$ higher than that of case 1 . When the twice of PV capacity is applied in case 5, the operation cost is greatly reduced, i.e., $\$ 148978$. The operation cost of both the first and second stages are lower among cases 1-5 when gas quality management is incorporated. In case 6, the lowest economic result is yielded without the gas quality ensured, the purchase
TABLE I

CASE ILLUSTRATION

\begin{tabular}{cccc}
\hline \hline $\begin{array}{c}\text { Case } \\
\text { No. }\end{array}$ & $\begin{array}{c}\text { PV system } \\
\text { capacity } \\
(\mathrm{kVA})\end{array}$ & $\begin{array}{c}\text { Gas system } \\
\text { connection }\end{array}$ & $\begin{array}{c}\text { Gas quality } \\
\text { management }\end{array}$ \\
\hline 1 & 400 & Yes & Yes \\
2 & 400 & P2G & Yes \\
3 & 400 & G2P & Yes \\
4 & 400 & No & Yes \\
5 & 800 & Yes & Yes \\
6 & 400 & Yes & No \\
\hline \hline
\end{tabular}
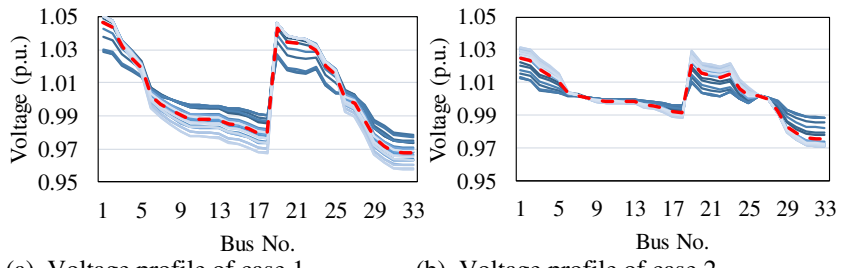

(a). Voltage profile of case 1 .

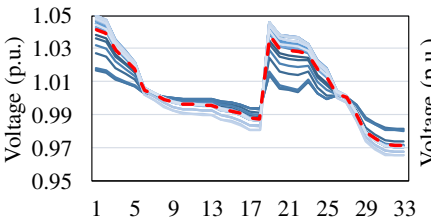

(b). Voltage profile of case 2

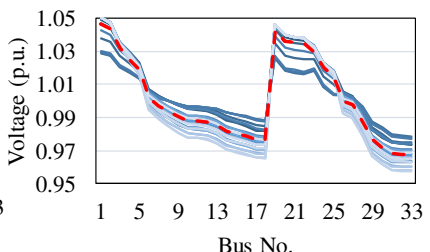

(c). Voltage profile of case 3 .

(d). Voltage profile of case 4 .

Fig. 4. Expected real-time voltage profiles.

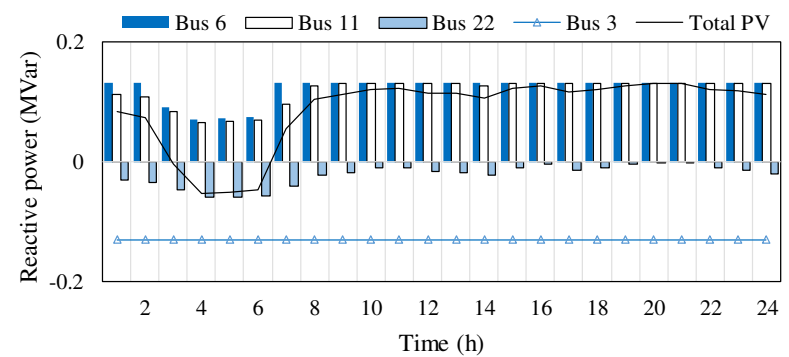

Fig. 5. Reactive power output of PV systems for case 1.

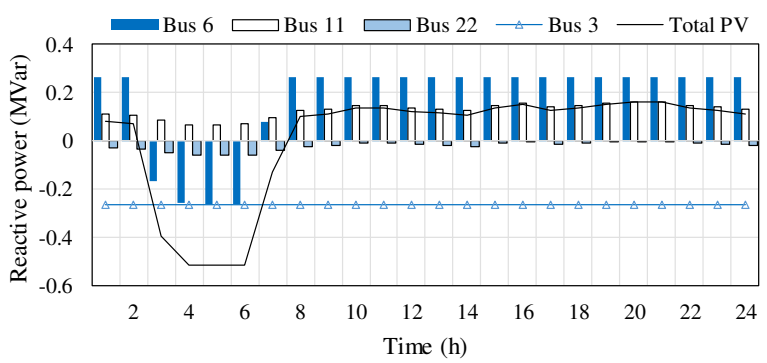

Fig. 6. Reactive power output of PV systems for case 5.

and injection cost of LPG and nitrogen is avoided, which results in $\$ 115896$ and $\$ 23176$ in the first and second stages, respectively.

Fig. 8 depicts the scheduling of energy hub connected with bus 8 and node 13. It can be seen that the usage of GF is the lowest. The reason is the conversion efficiency of GF is the lowest, which is considered as the backup conversion plan for replacing GSHP. GSHP is extensively used during the time period of 5:00-11:00, 12:00-16:00 and 18:00-24:00. In the morning, GSHP is injected by $1086 \mathrm{kWh}$ heat and peaks at $261 \mathrm{~kW}$, producing $3258 \mathrm{kWh}$ heat. This large amount of heat 


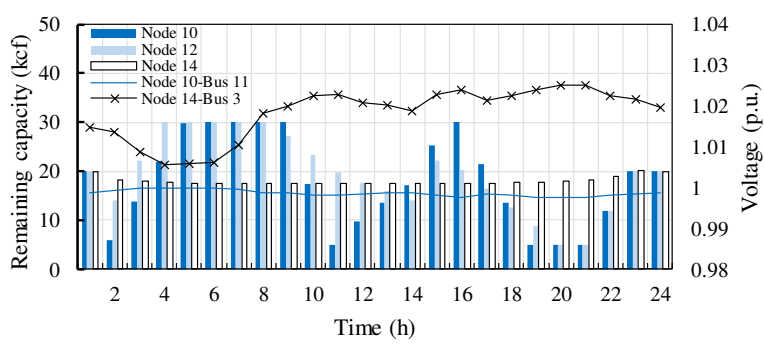

Fig. 7. Remaining capacity of DGS.

TABLE II

ECONOMIC PERFORMANCE FOR CASES 1-6

\begin{tabular}{lcccccc}
\hline \hline $\begin{array}{l}\text { Economic } \\
\text { result }\end{array}$ & Case 1 & Case 2 & Case 3 & Case 4 & Case 5 & Case 6 \\
\hline $\begin{array}{l}\text { First-stage } \\
\text { cost (\$) }\end{array}$ & 126244 & 134194 & 124298 & 124714 & 123232 & 115896 \\
$\begin{array}{l}\text { Expected } \\
\begin{array}{l}\text { Second-stage } \\
\text { cost (\$) }\end{array}\end{array}$ & 30458 & 32572 & 31608 & 32528 & 25746 & 23176 \\
\begin{tabular}{l} 
Total cost (\$) \\
\hline \hline
\end{tabular} & 156702 & 166766 & 155906 & 157242 & 148978 & 139072 \\
\hline \hline
\end{tabular}

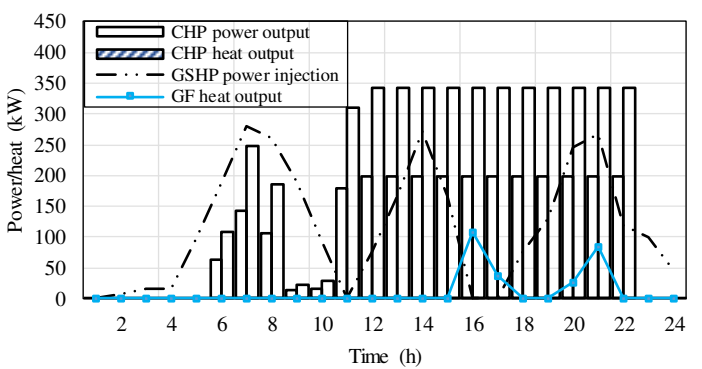

Fig. 8. Energy hub scheduling result.

energy can be consumed by heating load directly or stored by the heat storage system. During the period of 12:00-16:00, $677 \mathrm{kWh}$ is converted through GSHP. The last intensive conversion of GSHP is scheduled between 18:00 and 24:00, which results in $980 \mathrm{kWh}$ conversion from electricity to heat. Compared with GSHP, CHP yields $210 \%$ more energy conversion, particularly between 11:00 and 23:00. The gaspower conversion peaks at $198 \mathrm{~kW}$ and gas-heat peaks at $342 \mathrm{~kW}$. It is found that the gas-heat conversion is $82 \%$ higher than the gas-power conversion. The CHP converts gas to power/heat based on their corresponding efficiency and the gasheat conversion is higher than G2P.

\section{Studies on Gas Quality Management}

This subsection illustrates another main function of VPO, i.e., gas quality management. In Fig. 9, WI and SG are analysed for cases 1 and 6 . Case 1 considers gas quality management while case 6 does not. Generally, case 1 shows higher WI than case 6 but it remains within the allowable range (30-45). Without the gas quality constraints, case 6 violates the range, i.e., between 2:00 and 7:00, the WI of case 6 is below 30. Case 1 has a higher WI. And both the two indices are controlled at the permitted level.

The scheduling information of gas pressure under cases 1-3 for all the gas nodes is given in Fig. 10. Case 2 shows the highest gas pressure for all the nodes. The highest pressure is $28 \mathrm{Psig}$ at node 8 . The reason is that the additional power support from P2G facilities increases the gas flow and influences on the node pressure. Case 3 shows the minimum gas pressure which ranges

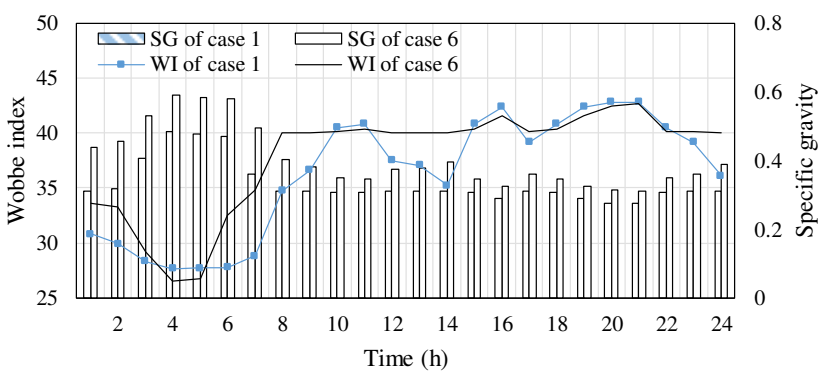

Fig. 9. Gas quality indices for cases 1 and 6.

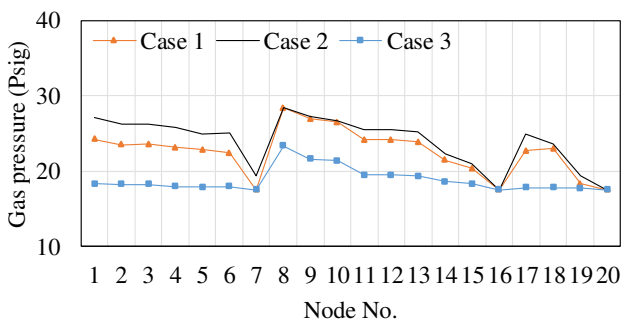

Fig. 10. Gas pressure for cases 1-3.

between 17 and 23 Psig. Since G2P provides excessive gas to the power system with the decrease of gas node pressure. Along the main branches in the gas system, i.e., nodes 1-7 and nodes $8-16$, the gas pressure peaks at nodes 1,8 and 17 . 8Since the pressure decreases with the reduction of gas flow.

\section{Scalability Analysis}

In this section, a scalability analysis is implemented on an IES with a larger scale, consisting of a modified IEEE 69-bus power system, two 20-node gas systems and 4 energy hubs. This system contains 6 PV systems, which are located at buses 9, 23, 26, 34, 44 and 58, respectively. The capacity of each PV system is $800 \mathrm{kVA}$. There are 12 capacitor banks for reactive power compensation and each capacity is $360 \mathrm{kVA}$. The two gas systems contain 4 P2G facilities and 4 DGSs. The following cases are considered to investigate the voltage-pressure joint management problem.

Case 1: Benchmark case.

Case 2: Applying twice the capacity of PV systems.

Case 3: Applying twice the capacity of capacitor banks.

Case 4: Applying twice the unit price of natural gas sources.

TABLE III shows the economic performance of all the cases. It can be seen that case 3 shows the lowest operation cost whilst case 4 shows the highest. In case 1, the first and second stage operation costs are $\$ 270288$ and $\$ 76571$, respectively. Compared with case 1, case 2 applied the twice capacity of PV systems, which yields $\$ 22260$ less operation cost. In case 3 , the doubled capacity of capacitor banks provides additional reactive power support, which is more effective in regulating the voltage magnitude. Therefore, less voltage deviation contributes to less operation cost. In case 4, the operation cost in the two stages is $\$ 379240$ and $\$ 75645$, respectively. The total operation cost is $31 \%$ higher than that of the benchmark case since twice the natural gas sources generation cost is considered

In Figs 11-13, the voltage profile of cases 1, 3 and 4 are given. In Fig. 11, the voltage magnitude ranges from 0.97 p.u. to 1.04 p.u.. When twice the capacity of capacitor banks is considered, the doubled reactive power support is effective on reducing the voltage fluctuation and magnitude. It can be seen that the 
TABLE III

ECONOMIC PERFORMANCE

\begin{tabular}{lcccc}
\hline \hline $\begin{array}{l}\text { Economic } \\
\text { result }\end{array}$ & Case 1 & Case 2 & Case 3 & Case 4 \\
\hline $\begin{array}{l}\text { First-stage } \\
\text { cost (\$) }\end{array}$ & 270288 & 262114 & 260578 & 379240 \\
$\begin{array}{l}\text { Expected } \\
\text { Second-stage } \\
\text { cost (\$) }\end{array}$ & 76571 & 62485 & 57930 & 75645 \\
\begin{tabular}{l} 
Total cost (\$) \\
\hline \hline
\end{tabular} & 346859 & 324599 & 318508 & 454885 \\
\hline
\end{tabular}

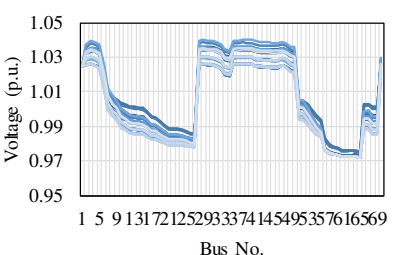

Fig. 11. Voltage profile of case 1.

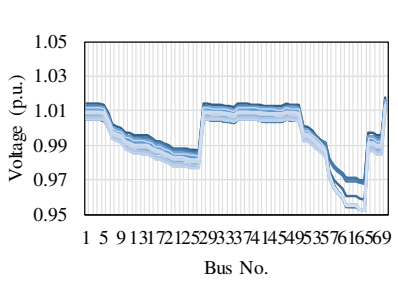

Fig. 13. Voltage profile of case 4.

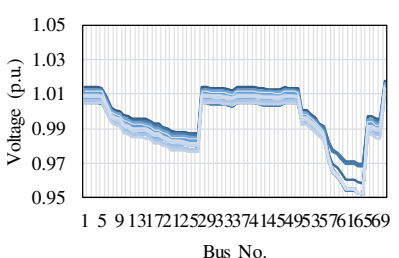

Fig. 12. Voltage profile of case 3 .

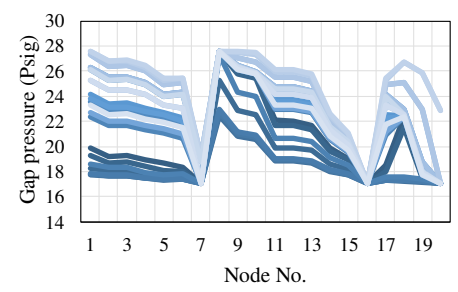

Fig. 14. Gas pressure profile.

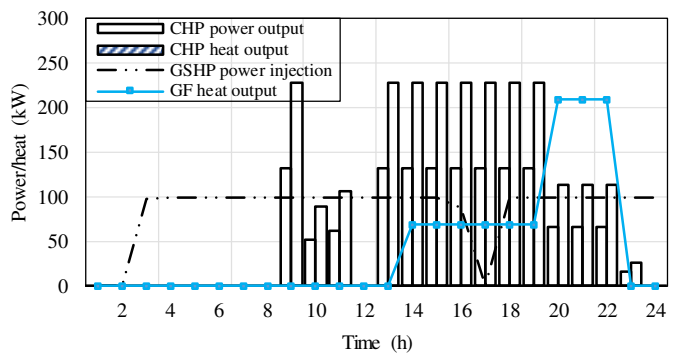

Fig. 15. Converter scheduling result of case 1.

voltage level of case 3 is between 0.95 p.u. and 1.02 p.u.. Moreover, the voltage fluctuation is greatly mitigated with respect to different solar generation at each hour. When twice the gas source unit cost is applied, the voltage profile shows the result between 0.97 and 1.03 p.u.. Since more power is injected into the gas system to ensure the overall economic performance of the system. The gas pressure profile is given in Fig. 14, which is between 17 and 28Psig. Compared with Fig. 10, Fig. 14 shows a similar gas pressure curve and magnitude. In Fig. 15, the energy hub scheduling is shown for the 69-bus test system. Compared with Fig. 8, CHP still dominates the energy conversion whilst GF results in higher power-heat conversion.

\section{E. Result Discussion}

This section is used to summarize the findings of the case studies. For the test in 33-bus-20-node IES, 6 cases are considered to testify the impact of gas system connection, PV system capacity and gas quality management on the results of voltage profile, economic performance and gas pressure profile. The results demonstrate that without gas quality management, the operation cost is the lowest. However, this operation scheme sacrifices the gas quality, which will inevitably lead to quality issues of gas distribution and customer usage. When the G2P is disconnected, the operation cost is $6 \%$ higher than that of the benchmark case. Meanwhile, the voltage fluctuation is relatively moderate compared with other cases. In case 4, without the gas connection, the voltage profile shows a similar result compared with the benchmark case, which indicates that the bidirectional gas interconnection has a minor impact on the voltage profile. The gas pressure result shows the unidirectional energy flow, i.e., P2G, leads to a relatively higher gas pressure profile. In addition, the gas quality results imply the necessity of incorporating a gas quality management scheme in the IES operation. The scalability analysis is conducted on a 69-bus power system connected with two 20-node gas system and 4 energy hubs. The capacity supplement on capacitor banks results in higher economic efficiency than that on PV systems. The voltage profile results demonstrate that effective voltage regulation when applying twice the capacity of capacitor banks. In addition, the higher generation cost of gas sources also contributes to voltage regulation. Gas pressure profile shows a similar result compared with the small-scale 33-bus IES.

Future work aims to i) investigate a multi timescale voltage management framework to differentiate slow-response and fastresponse voltage regulating devices for more accurate voltage regulation and ii) consider time delay in the model for better practicability.

\section{CONCLUSION}

In this paper, a two-stage VPO model is developed to successfully regulate voltage deviation, manage gas quality and minimize system operation cost for IESs. P2G facilities and GSS are innovatively utilized as voltage regulating devices for converting and storing surplus renewable power generation. A gas quality management mechanism is developed to handle the hydrogen injection for ensuring the secure and economic operation of the gas system. The TS-VPO model provides a day-ahead preparation and real-time adaptive operation scheme. The ambiguity set employs the Wasserstein metric to capture all the possible candidate distributions. LDR is applied for approximating the recourse decisions. From the extensive case studies, the proposed VPO successfully facilitates the efficiency and economy of IES operation in regards to the voltage magnitude regulation and gas pressure control with minimized operation cost. Furthermore, it contributes to a reliable and sustainable energy supply to end customers and the society under the era of multi-energy with high renewable penetration.

\section{REFERENCES}

[1]S. Li, H. He, C. Su, and P. Zhao, "Data driven battery modeling and management method with aging phenomenon considered," Applied Energy, vol. 275, p. 115340, 2020/10/01/ 2020, doi: https://doi.org/10.1016/j.apenergy.2020.115340.

[2] S. Li, H. He, and J. Li, "Big data driven lithium-ion battery modeling method based on SDAE-ELM algorithm and data pre-processing technology," Applied Energy, vol. 242, pp. 1259-1273, 2019/05/15/ 2019, doi: https://doi.org/10.1016/j.apenergy.2019.03.154.

[3]X. Xu, Y. Jia, Y. Xu, Z. Xu, S. Chai, and C. S. Lai, "A Multi-agent Reinforcement Learning based Data-driven Method for Home Energy Management," IEEE Transactions on Smart Grid, pp. 1-1, 2020, doi: 10.1109/TSG.2020.2971427.

[4]H. R. Baghaee, M. Mirsalim, G. B. Gharehpetian, and A. K. Kaviani, "Security/cost-based optimal allocation of multi-type FACTS devices using 
multi-objective particle swarm optimization," SIMULATION, vol. 88, no. 8, pp. 999-1010, 2012/08/01 2012, doi: 10.1177/0037549712438715.

[5]H. Baghaee, M. Mirsalim, and G. B. Gharehpetian, "Multi-objective optimal power management and sizing of a reliable wind/PV microgrid with hydrogen energy storage using MOPSO," Journal of Intelligent \& Fuzzy Systems, vol. 32, pp. 1753-1773, 02/24 2017, doi: 10.3233/JIFS-152372.

[6]H. R. Baghaee, M. Mirsalim, G. B. Gharehpetian, and H. A. Talebi, "MOPSO/FDMT-based Pareto-optimal solution for coordination of overcurrent relays in interconnected networks and multi-DER microgrids," IET Generation, Transmission \& Distribution, vol. 12, no. 12, pp. 2871-2886, 2018, doi: 10.1049/iet-gtd.2018.0079.

[7]A. Parizad and K. Hatziadoniu, "Security/stability-based Pareto optimal solution for distribution networks planning implementing NSGAII/FDMT," Energy, vol. 192, p. 116644, 2020/02/01/ 2020, doi: https://doi.org/10.1016/j.energy.2019.116644.

[8]Y. Chen et al., "Robust N-k CCUC model considering the fault outage probability of units and transmission lines," IET Generation, Transmission \& Distribution, vol. 13, no. 17, pp. 3782-3791, 2019, doi: 10.1049/ietgtd.2019.0780.

[9]Y. Chen, Z. Zhang, H. Chen, and H. Zheng, "Robust UC model based on multi-band uncertainty set considering the temporal correlation of wind/load prediction errors," IET Generation, Transmission \& Distribution, vol. 14, no. 2, pp. 180-190, 2020, doi: 10.1049/iet-gtd.2019.1439.

[10] H. Baghaee, M. Mirsalim, G. B. Gharehpetian, and A. Talebi, "Generalized three phase robust load-flow for radial and meshed power systems with and without uncertainty in energy resources using dynamic radial basis functions neural networks," Journal of Cleaner Production, vol. 174, pp. 96113, 02/10 2018, doi: 10.1016/j.jclepro.2017.10.316.

[11] H. R. Baghaee, M. Mirsalim, G. B. Gharehpetian, and H. A. Talebi, "Three-phase AC/DC power-flow for balanced/unbalanced microgrids including wind/solar, droop-controlled and electronically-coupled distributed energy resources using radial basis function neural networks," IET Power Electronics, vol. 10, no. 3, pp. 313-328, 2017, doi: 10.1049/iet-pel.2016.0010. [12] Y. Xu, Z. Y. Dong, R. Zhang, and D. J. Hill, "Multi-Timescale Coordinated Voltage/Var Control of High Renewable-Penetrated Distribution Systems," IEEE Transactions on Power Systems, vol. 32, no. 6, pp. 4398-4408, 2017, doi: 10.1109/TPWRS.2017.2669343.

[13] Q. Zhang, K. Dehghanpour, and Z. Wang, "Distributed CVR in Unbalanced Distribution Systems With PV Penetration," IEEE Transactions on Smart Grid, vol. 10, no. 5, pp. 5308-5319, 2019, doi: 10.1109/TSG.2018.2880419.

[14] P. Zhao, C. Gu, D. Huo, Y. Shen, and I. Hernando-Gil, "Two-Stage Distributionally Robust Optimization for Energy Hub Systems," IEEE Transactions on Industrial Informatics, vol. 16, no. 5, pp. 3460-3469, 2020, doi: 10.1109/TII.2019.2938444.

[15] P. Zhao, C. Gu, Y. Xiang, X. Zhang, Y. Shen, and S. Li, "Reactive Power Optimization in Integrated Electricity and Gas Systems," IEEE Systems Journal, pp. 1-11, 2020, doi: 10.1109/JSYST.2020.2992583.

[16] E. A. M. Ceseña and P. Mancarella, "Energy Systems Integration in Smart Districts: Robust Optimisation of Multi-Energy Flows in Integrated Electricity, Heat and Gas Networks," IEEE Transactions on Smart Grid, vol. 10, no. 1, pp. 1122-1131, 2019, doi: 10.1109/TSG.2018.2828146.

[17] Y. Wang et al., "Optimal Scheduling of the Regional Integrated Energy System Considering Economy and Environment," IEEE Transactions on Sustainable Energy, vol. 10, no. 4, pp. 1939-1949, 2019, doi: 10.1109/TSTE.2018.2876498.

[18] N. Liu, J. Wang, and L. Wang, "Hybrid Energy Sharing for Multiple Microgrids in an Integrated Heat-Electricity Energy System," IEEE Transactions on Sustainable Energy, vol. 10, no. 3, pp. 1139-1151, 2019, doi: 10.1109/TSTE.2018.2861986.

[19] Y. Li, Z. Li, F. Wen, and M. Shahidehpour, "Privacy-Preserving Optimal Dispatch for an Integrated Power Distribution and Natural Gas System in Networked Energy Hubs," IEEE Transactions on Sustainable Energy, vol. 10, no. 4, pp. 2028-2038, 2019, doi: 10.1109/TSTE.2018.2877586.

[20] Y. Cheng, N. Zhang, B. Zhang, C. Kang, W. Xi, and M. Feng, "LowCarbon Operation of Multiple Energy Systems Based on Energy-Carbon Integrated Prices," IEEE Transactions on Smart Grid, pp. 1-1, 2019, doi: 10.1109/TSG.2019.2935736.

[21] X. Xing, J. Lin, Y. Song, and Q. Hu, "Maximum Production Point Tracking of a High-Temperature Power-to-Gas System: A Dynamic-ModelBased Study," IEEE Transactions on Sustainable Energy, vol. 11, no. 1, pp. 361-370, 2020, doi: 10.1109/TSTE.2019.2891296.

[22] Y. Li et al., "Optimal Stochastic Operation of Integrated Low-Carbon Electric Power, Natural Gas, and Heat Delivery System," IEEE Transactions on
Sustainable Energy, vol. 9, no. 1, pp. 273-283, 2018, doi: 10.1109/TSTE.2017.2728098.

[23] Y. Li, W. Liu, M. Shahidehpour, F. Wen, K. Wang, and Y. Huang, "Optimal Operation Strategy for Integrated Natural Gas Generating Unit and Power-to-Gas Conversion Facilities," IEEE Transactions on Sustainable Energy, vol. 9, no. 4, pp. 1870-1879, 2018, doi: 10.1109/TSTE.2018.2818133. [24] I. Union, "Petroleum B. guidebook to gas interchangeability and gas quality," 2011.

[25] I. A. Gondal, "Hydrogen integration in power-to-gas networks," International Journal of Hydrogen Energy, vol. 44, no. 3, pp. 1803-1815, 2019/01/15/ 2019, doi: https://doi.org/10.1016/j.ijhydene.2018.11.164.

[26] L. de Santoli, G. Lo Basso, and D. Bruschi, "A small scale H2NG production plant in Italy: Techno-economic feasibility analysis and costs associated with carbon avoidance," International Journal of Hydrogen Energy, vol. 39, no. 12, pp. 6497-6517, 2014/04/15/ 2014, doi: https://doi.org/10.1016/j.ijhydene.2014.02.003.

[27] P. Zhao, C. Gu, and D. Huo, "Two-Stage Coordinated Risk Mitigation Strategy for Integrated Electricity and Gas Systems under Malicious False Data Injections," IEEE Transactions on Power Systems, pp. 1-1, 2020, doi: 10.1109/TPWRS.2020.2986455.

[28] X. Lu, K. W. Chan, S. Xia, X. Zhang, G. Wang, and F. Li, "A Model to Mitigate Forecast Uncertainties in Distribution Systems Using the Temporal Flexibility of EVAs," IEEE Transactions on Power Systems, vol. 35, no. 3, pp. 2212-2221, 2020, doi: 10.1109/TPWRS.2019.2951108.

[29] P. Zhao, C. Gu, Z. Hu, X. I. E. D, I. Hernando-Gil, and Y. Shen, "Distributionally Robust Hydrogen Optimization with Ensured Security and Multi-Energy Couplings," IEEE Transactions on Power Systems, pp. 1-1, 2020, doi: 10.1109/TPWRS.2020.3005991.

[30] X. Lu, K. W. Chan, S. Xia, B. Zhou, and X. Luo, "Security-Constrained Multiperiod Economic Dispatch With Renewable Energy Utilizing Distributionally Robust Optimization," IEEE Transactions on Sustainable Energy, vol. 10, no. 2, pp. 768-779, 2019, doi: 10.1109/TSTE.2018.2847419.

[31] B. Zhang, A. Y. S. Lam, A. D. Domínguez-García, and D. Tse, "An Optimal and Distributed Method for Voltage Regulation in Power Distribution Systems," IEEE Transactions on Power Systems, vol. 30, no. 4, pp. 1714-1726, 2015, doi: 10.1109/TPWRS.2014.2347281.

[32] P. S. Roy, C. Ryu, and C. S. Park, "Predicting Wobbe Index and methane number of a renewable natural gas by the measurement of simple physical properties," Fuel, vol. 224, pp. 121-127, 2018/07/15/ 2018, doi: https://doi.org/10.1016/j.fuel.2018.03.074

[33] Z. Hu and X. Zhang, "Study on laminar combustion characteristic of low calorific value gas blended with hydrogen in a constant volume combustion bomb," International Journal of Hydrogen Energy, vol. 44, no. 1, pp. 487-493, 2019/01/01/ 2019, doi: https://doi.org/10.1016/j.ijhydene.2018.02.055.

[34] L. Kong, L. Su, X. Zhou, L. Chen, and Q. Liu, "Accuracy guarantee for determining the calorific value for dual gas sources," Flow Measurement and Instrumentation, vol. 65, pp. 233-239, 2019/03/01/ 2019, doi: https://doi.org/10.1016/j.flowmeasinst.2019.01.003.

[35] M. Deymi-Dashtebayaz, A. Ebrahimi-Moghadam, S. I. Pishbin, and M. Pourramezan, "Investigating the effect of hydrogen injection on natural gas thermo-physical properties with various compositions," Energy, vol. 167, pp. 235-245, 2019/01/15/ 2019, doi: https://doi.org/10.1016/j.energy.2018.10.186. [36] L. Xiang, G. Theotokatos, and Y. Ding, "Investigation on gaseous fuels interchangeability with an extended zero-dimensional engine model," Energy Conversion and Management, vol. 183, pp. 500-514, 2019/03/01/ 2019, doi: https://doi.org/10.1016/j.enconman.2019.01.013.

[37] H. Yeh, D. F. Gayme, and S. H. Low, "Adaptive VAR Control for Distribution Circuits With Photovoltaic Generators," IEEE Transactions on Power Systems, vol. 27, no. 3, pp. 1656-1663, 2012, doi: 10.1109/TPWRS.2012.2183151.

[38] B. Wang, C. Zhang, and Z. Dong, "Interval Optimization Based Coordination of Demand Response and Battery Energy Storage System Considering SoC Management in A Microgrid," IEEE Transactions on Sustainable Energy, pp. 1-1, 2020, doi: 10.1109/TSTE.2020.2982205.

[39] S. Doan, H. Yeh, and Y. Yang, "Two-Mode Adaptive Schemes for VAR Control With Solar Power and Energy Storage," IEEE Systems Journal, vol. 14, no. 1, pp. 889-899, 2020, doi: 10.1109/JSYST.2019.2920016.

[40] J. Qiu, J. Zhao, H. Yang, D. Wang, and Z. Y. Dong, "Planning of solar photovoltaics, battery energy storage system and gas micro turbine for coupled micro energy grids," Applied Energy, vol. 219, pp. 361-369, 2018/06/01/ 2018, doi: https://doi.org/10.1016/j.apenergy.2017.09.066.

[41] P. M. Esfahani and D. Kuhn, "Data-driven distributionally robust optimization using the Wasserstein metric: Performance guarantees and tractable reformulations," Mathematical Programming, vol. 171, no. 1-2, pp. 115-166, 2018. 
[42] D. Bertsimas, X. V. Doan, K. Natarajan, and C.-P. Teo, "Models for minimax stochastic linear optimization problems with risk aversion," Mathematics of Operations Research, vol. 35, no. 3, pp. 580-602, 2010.

[43] A. Ben-Tal, A. Goryashko, E. Guslitzer, and A. Nemirovski, "Adjustable robust solutions of uncertain linear programs," Mathematical programming, vol. 99, no. 2, pp. 351-376, 2004.

[44] A. Shapiro, "On duality theory of conic linear problems," in Semi-infinite programming: Springer, 2001, pp. 135-165.

[45] I. I. o. T. Electrical and Computer Engineering Department. "Index of /data." motor.ece.iit.edu/data/ (accessed.

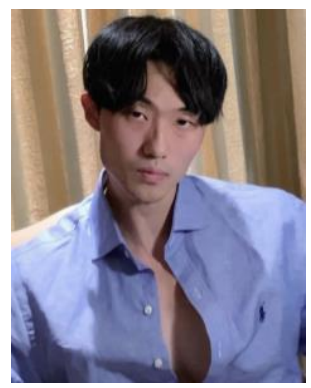

Pengfei Zhao (S'18) was born in Beijing, China. $\mathrm{He}$ received the double B.Eng. degree from the University of Bath, U.K., and North China Electric Power University, Baoding, China, in 2017. He is currently pursuing the Ph.D. degree with the Department of Electronic and Electrical Engineering, University of Bath, U.K. He was a visiting Ph.D. student at Smart Grid Operations and Optimization Laboratory (SGOOL), Tsinghua University, Beijing, China in 2019. His major research interests include the operation and planning of integrated energy systems considering

inherent uncertainties.

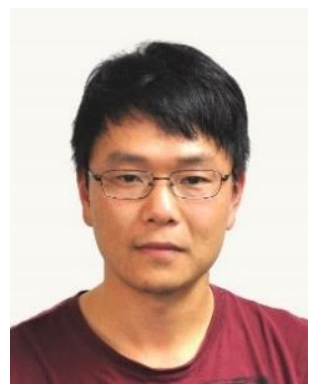

Chenghong Gu (M'14) was born in Anhui province, China. He received the Master's degree from the Shanghai Jiao Tong University, Shanghai, China, in 2007 in electrical engineering. He received the Ph.D. degree from the University of Bath, U.K. He is currently a Lecturer and EPSRC Fellow with the Department of Electronic and Electrical Engineering, University of Bath. His major research interest is in multi-vector energy system, smart grid, and power economics.

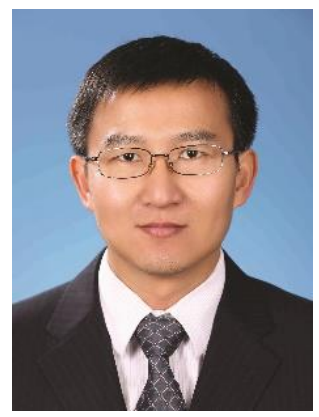

Zechun Hu (M'09-SM'17) received the B.S. and $\mathrm{Ph} . \mathrm{D}$. degrees from Department of Electrical Engineering, Xi'an Jiao Tong University, Xi'an, China, in 2000 and 2006, respectively. He worked in Shanghai Jiao Tong University after graduation and also worked in University of Bath as a research officer from 2009 to 2010. He joined the Department of Electrical Engineering at Tsinghua University in 2010 where he is now an associate professor. He has published more than 150 peer-reviewed papers and two books. He serves as an associate editor of IEEE Transactions on Transportation Electrification. His major research interests include vehicle to grid techniques, applications of energy storage in power systems, optimal planning and operation of power systems, and electricity markets.

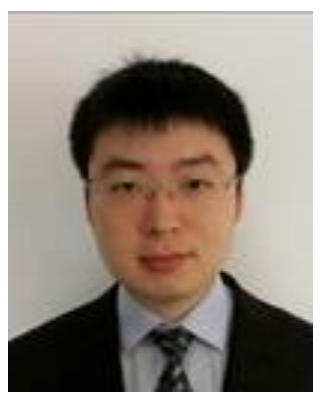

Xin Zhang received his B.Eng. degree in automation from Shandong University, China, in 2006; his M.S. and Ph.D. degrees in electrical power engineering from The University of Manchester, U.K., in 2007 and 2010 respectively. $\mathrm{He}$ is a senior lecturer in energy systems at Cranfield University, U.K. He previously worked for electricity national control at the National Grid, U.K. His main research interests include power system operation, renewable energy integration, and low carbon technologies in energy systems.

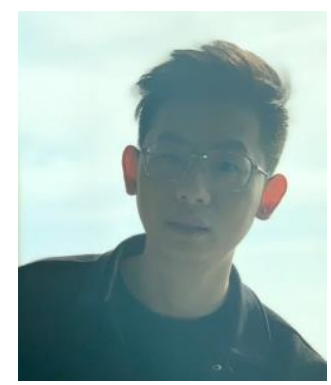

Xinlei Chen is currently a postdoctoral research associate in Electrical Engineering Department at Carnegie Mellon University. He received the B.E. and M.S. degrees in Electronic Engineering from Tsinghua University, China, in 2009 and 2012, respectively, and Ph.D degrees in Electrical Engineering from Carnegie Mellon University, Pittsburgh, PA, USA. His research interests lie in mobile computing, crowd intelligence, cyber physical system, mobile embedded system etc.

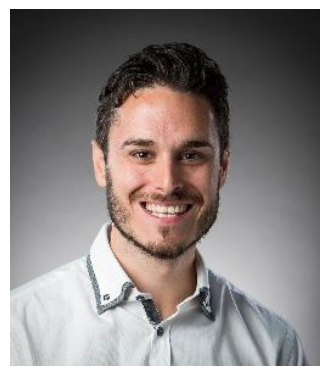

Ignacio Hernando-Gil (S'10-M'14) received the Ph.D. degree in power systems from the University of Edinburgh, U.K., in 2014. He is currently Associate Professor at ESTIA Institute of Technology, France, and was previously Prize Fellow at the University of Bath, U.K., and Research Fellow at the University of Edinburgh, U.K. He was also in industry with PassivSystems Ltd., U.K., and National Grid U.K. He has extensive research in risk modelling and analysis of active distribution networks and the aggregate of power supply. impact of smart grid technologies on the quality

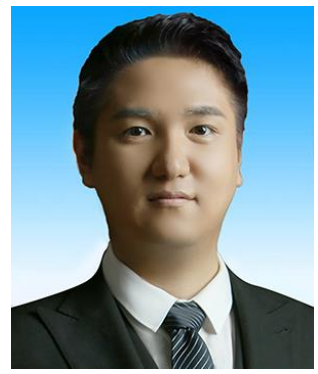

Yucheng Ding was born in China in 1990. He received the B.S. degree in electronic and information engineering and the M.S. degree in control theory and control engineering both from Liaoning Technical University, Liaoning, China, in 2013 and 2016, respectively. He is currently pursuing Ph.D. degree at China Electric Power Research Institute. His research interests include power system security and stability, AI applications in power systems. 